\title{
LOS CONTRATOS SOCIALES: ¿UN NUEVO FORMALISMO ÉTICO?
}

Social contracts: a new ethical formalism?

Miguel López Astorga*

\section{Resumen}

La teoría de los contratos sociales sostiene que existen mecanismos evolutivos y reglas adaptativas para regular los intercambios sociales. En este trabajo analizamos dos experimentos que esta teoría ha presentado con el propósito de apoyar sus hipótesis. Después de tal revisión, llegamos a la conclusión de que los supuestos fundamentales de la teoría de los contratos sociales no quedan demostrados y de que sus resultados experimentales pueden ser explicados por medio de la lógica formal clásica.

Palabras clave: Contratos sociales, mecanismos evolutivos, reglas adaptativas, inferencia lógica.

Abstract

Social contracts theory holds that evolutionary mechanisms and adaptative rules regulate social exchanges. In this paper, we analyze two experiments that this theory has presented for supporting its hypotheses. After such a review, we conclude that fundamental suppositions of social contracts theory are not demonstrated and that its experimental outcomes can be explained from formal classic logic.

Key words: Social contracts, adaptative mechanisms, adaptative rules, logical inference.

\section{A Modo DE INTRODUCCIÓN: AVANCES PREVIOS}

Aunque la teoría de los contratos sociales ya ha sido revisada $y$ cuestionada por nosotros (López Astorga, 2004), ${ }^{1}$ puesto que se trata de un

\footnotetext{
${ }^{1}$ Este artículo es el resultado de un proceso de investigación comenzado años atrás y, por tanto, se apoya en los resultados y en las conclusiones de trabajos anteriores. Nuestro propósito es presentar aquí un texto autocontenido que permita, en la medida de lo posible, una lectura autónoma. Sin embargo, pensamos que no podemos comenzar sin hacer algunas precisiones. Nos basamos en nuestras exposiciones y argumentaciones en la descripción de la teoría de los contratos sociales y en la traducción del experimento 1 de Fiddick, Cosmides y Tooby (2000) que ofrecimos en López Astorga (2004). Del mismo modo, las tesis de Almor y Sloman (2000) que comentamos más abajo ya fueron analizadas por nosotros en López Astorga (2008) trabajo, este último, en el que también explicamos las líneas generales de la tarea de selección de las cuatro tarjetas de Peter Wason a las que vamos a hacer referencia en los apartados en los que va a ser necesario. Aclarados estos puntos, podemos decir que lo novedoso de este artículo — que procede directamente de los textos mencionados e indirectamente de otros no citados- no consiste en evidenciar los supuestos con apoyos débiles y los aspectos cuestionables que pueden detectarse en Fiddick, Cosmides y Tooby (2000); en Gigerenzer y Hug (1992) — lo cual
} 


\section{Miguel López Astorga}

enfoque cognitivo desde el que se sigue trabajando y diseñando experimentos, consideramos totalmente justificado continuar profundizando en el análisis de sus tesis fundamentales y de los apoyos empíricos en los que trata de apoyarse. En nuestra opinión, por una parte, sus tesis no quedan demostradas a partir de sus resultados experimentales y, por otra, tales resultados son interpretables a partir de otros marcos teóricos. Ya que son bastante numerosos los trabajos en los que se argumenta a favor de los contratos sociales, hemos seleccionado, para examinar a lo largo de estas páginas, dos problemas representativos que, según sus defensores, prueban la validez de la mencionada teoría. Estos dos problemas son el que se puede encontrar en el experimento 1 de Fiddick, Cosmides y Tooby (2000) y el problema de la cabaña de Gigerenzer y Hug (1992).

Con respecto al experimento 1 de Fiddick, Cosmides y Tooby (2000), ya avanzamos (López Astorga, 2004) que, aparentemente, se presentó con el propósito de refutar las tesis de la teoría de la relevancia defendida por Sperber, Cara y Girotto (1995). En López Astorga (2004), recurrimos al enfoque de Almor y Sloman (2000) con el objetivo de poner de manifiesto que el comportamiento de los sujetos experimentales de Fiddick, Cosmides y Tooby podía no deberse a la acción de mecanismos inferenciales específicos como los defendidos en su propuesta, sino al procesamiento de la información contenida en las instrucciones de su experimento 1 que podían realizar tales sujetos. En este nuevo trabajo que ahora presentamos, volvemos a apelar a las teorías de Almor y Sloman (2000) para defender que la lógica formal clásica, sin la ayuda de ningún otro recurso, puede explicar los resultados del experimento 1 de Fiddick, Cosmides y Tooby (2000).

En cuanto al problema de la cabaña de Gigerenzer y Hug (1992), ya establecimos (López Astorga, 2004) que es controvertido, puesto que sus dos condiciones experimentales no son verdaderamente comparables. Una de ellas implica menos esfuerzo por parte del sujeto para interpretar sus instrucciones. En el apartado dedicado a este problema, profundizamos aún más y, como hemos indicado, hacemos notar que la condición más compleja no sólo va acompañada de una mayor dificultad, sino que es una versión de la tarea de selección que es imposible de solucionar y que, por tanto, carece, desde nuestro punto de vista, de valor explicativo.

ya ha sido puesto de relieve por nosotros en López Astorga (2004) mediante las investigaciones de Almor y Sloman (2000) — sino en demostrar que los resultados del experimento 1 de Fiddick, Cosmides y Tooby (2000) son explicables a partir del marco teórico de la lógica proposicional y que el problema de la cabaña de Gigerenzer y Hug (1992) no es una buena tarea para analizar el razonamiento humano, pues, al no disponer de una clara respuesta correcta, no puede ser resuelta en sentido lógico estricto. 
Así, proponemos, a continuación, un texto que pretende ser una contribución para el estudio de la mente y del razonamiento humano y acercarse a la posibilidad de determinar cuál es el verdadero estatus gnoseológico de las reglas y los principios lógicos.

\section{LA NUEVA TEORÍA DEL CONTRATO SOCIAL}

Con la expresión "teoría de los contratos sociales" — relativa, en la época contemporánea, a la hipótesis de la existencia de mecanismos evolutivos y reglas adaptativas para regular los intercambios sociales - no vamos a hacer referencia, aquí, a las propuestas de la filosofía social y política que se remontan a planteamientos que suelen considerarse como representativos del llamado "contractualismo clásico" y que bien pueden tener su origen en el pensamiento de autores de la Ilustración como, por ejemplo, Rousseau u Hobbes. Lo que pretendemos analizar en este trabajo es una teoría que, utilizando una denominación semejante, procede de la psicología del razonamiento y de la ciencia cognitiva. Podemos encontrar una revisión interesante de este enfoque, por ejemplo, en Santamaría (1995). No obstante, si queremos centrarnos en textos originales de sus defensores, lo más aconsejable, quizás, es recurrir a artículos como el de Cosmides (1989).

Basándonos en los textos citados, podemos decir que la teoría de los contratos sociales defiende que los seres humanos contamos con reglas filogenéticas y mecanismos adaptativos que la selección natural nos ha proporcionado en los momentos finales de nuestra evolución (principalmente, a lo largo del pleistoceno). Según esta teoría, no es posible mantener la cultura sin intercambios sociales en los que cada sujeto perteneciente a la sociedad entregue beneficios y los consiga a través de sus interacciones con los demás individuos. De esta manera, para que la sociedad funcione correctamente, los intercambios deben realizarse de la manera oportuna, lo que implica, necesariamente, disponer de los recursos apropiados para poder detectar a los sujetos que no cumplen los acuerdos. Desde este enfoque teórico, contamos realmente con tales recursos, los cuales se manifiestan, de manera fundamental, en dos reglas

A.- Si una persona acepta el beneficio, entonces paga el costo.

B.- Si una persona paga el costo, entonces acepta el beneficio.

Existen más trabajos en los que se argumenta a favor de esta teoría. En concreto, Fiddick, Cosmides y Tooby (2000) creen que, durante los años noventa, surgió una propuesta teórica significativa, la de la racionalidad ecológica. ${ }^{2}$ A su juicio, son diversos los autores que sostienen este enfoque y

\footnotetext{
${ }^{2}$ Esta propuesta puede remontarse, según Fiddick, Cosmides y Tooby (2000) a trabajos como
} 


\section{Miguel López Astorga}

tienen en común el estar de acuerdo en que la mente humana cuenta con facultades cognitivas de contenido específico cuya función es realizar inferencias relacionadas con dicho contenido. Estas inferencias, así, complementan a las capacidades racionales generales de los seres humanos. De hecho, Fiddick, Cosmides y Tooby (2000) están convencidos de que la neurociencia cognitiva y la psicología evolutiva nos proporcionan pruebas en el momento presente acerca de mecanismos inferenciales autónomos referentes a ámbitos particulares, como el de la causalidad física, el de los números, el del mundo biológico, el de las creencias y motivaciones de otros individuos, el del control de los riesgos, el de las interacciones sociales y uno de esos mecanismos inferenciales puede ser, claro está, el recogido en la teoría de los contratos sociales.

Este planteamiento psicológico tiene, sin duda, en nuestra opinión, importantes implicaciones filosóficas y trascendentes consecuencias éticas. Desde nuestro punto de vista, el enfoque de los contratos sociales se asocia necesariamente con una visión muy particular de la naturaleza humana. Estaríamos ante individuos diseñados, evolutivamente, para controlar el orden social y para detectar incumplidores de las reglas. De ello, deducimos que, desde la visión de los defensores de los contratos sociales, el desarrollo social y el progreso de la civilización han sido únicamente posibles gracias a la aparición, en un momento determinado de la evolución, de sistemas y mecanismos regulativos de la dinámica social. Por otra parte, se ofrece una nueva versión de lo que, en filosofía práctica, se ha venido denominando, a partir de la época moderna, formalismo ético. Obviamente, no se trata de un formalismo en el sentido kantiano del término, pero sí de una teoría que habla de unas estructuras de pensamiento ético-social que son, por utilizar una expresión kantiana, a priori y que se aplican a contenidos procedentes del exterior. Dicho de otro modo, disponemos de estructuras mentales de control del comportamiento ajeno que deben recibir contenido a partir del entorno social en el que nos encontremos, pues este que nos indica cuáles son las obligaciones que tenemos que cumplir y a cambio de qué beneficios. No estamos ante un universalismo o un absolutismo éticos --ya que, según interpretamos, los beneficios, los costos y las obligaciones dependen del contexto sociocultural concreto- pero sí ante una teoría que sostiene que poseemos unos mecanismos mentales universales que controlan y dirigen la dinámica de las culturas y de los pueblos.

los de Barkow, Cosmides y Tooby (1992); Cosmides y Tooby (1996-1996b); Gigerenzer, Hoffrage y Kleinbolting (1991); Gigerenzer y Hoffrage (1995); Gigerenzer, Todd et al. (1999) y Sperber (1994). 
Así, consideramos que los defensores de los contratos sociales trascienden el plano psicológico y el cognitivo y se sitúan en el ámbito social, estableciendo, del mismo modo, un puente de tránsito que comunica al pensamiento lógico con la valoración ética. No obstante, como ya hemos señalado, ésta no es una propuesta filosófica más, sino un planteamiento que surge desde otras disciplinas y que pretende no ser solamente el producto de una especulación teórica, sino contar con apoyo experimental de corte científico. Puesto que las consecuencias que se derivan de esta teoría, en lo que respecta al concepto de ser humano, son realmente importantes, creemos justificado revisar algunos de los experimentos que, según los partidarios de este enfoque, demuestran la existencia de estos mecanismos especializados de intercambio social. En la literatura de la psicología cognitiva, podemos hallar un número significativo de tales experimentos. Empero, vamos a centrarnos inicialmente en uno que consideramos emblemático y lo suficientemente representativo: el experimento 1 de Fiddick, Cosmides y Tooby (2000), en el que se cuenta la historia de un granjero sudamericano al que le ofrecen maíz a cambio de papas.

\section{EL EXPERIMENTO DE LAS PAPAS Y EL MAÍZ}

La experimentación de Fiddick, Cosmides y Tooby (2000) se apoya en versiones de la tarea de selección de las cuatro tarjetas de Peter Wason (en adelante, vamos a referirnos a esta tarea con las siglas WST, procedentes de la expresión en lengua inglesa Wason Selection Task). Para comprender verdaderamente tal tarea, su significado y sus implicaciones recomendamos al lector acudir a sus fuentes originales, por ejemplo, a Wason (1966). No obstante, hemos de tener en cuenta que el problema de las papas y el maíz que Fiddick, Cosmides y Tooby (2000) proponen a sus sujetos experimentales presenta características adicionales y, por este motivo, lo reproducimos a continuación.

En una primera versión, la versión condicional, al participante se le solicita que suponga que es un granjero sudamericano que obtiene un excedente de papas al finalizar su cosecha. Por ello, viaja a una aldea cercana con la intención de obtener provecho de esta situación. En la aldea conoce a cuatro personas, con las que entabla una conversación, pero hay una dificultad: hablan un dialecto que el granjero no comprende totalmente. Él llega a entender únicamente una expresión semejante a ésta: "Si me da algunas papas, le daré un poco de maíz".

Así, se le muestran al sujeto cuatro tarjetas. Cada una de ellas hace referencia a uno de los aldeanos que conoció. Por un lado, cada tarjeta muestra si el granjero le dio o no papas a ese aldeano y, por otro, si ese mismo aldeano le correspondió con maíz o no. En las caras que él puede ver figuran, 
respectivamente: "Usted le dio papas a esta persona", "Usted no le dio papas a esta persona", "Esta persona le dio maíz a usted" y "Esta persona no le dio a usted nada".

La tarea del sujeto experimental es, entonces, seleccionar solamente aquella(s) tarjeta(s) necesaria(s) para comprobar si alguno de los aldeanos no ha cumplido con su parte del trato. La respuesta correcta es obvia. Si atendemos a la regla A del apartado anterior, tenemos que la primera tarjeta, "Usted le dio papas a esta persona", debe ser levantada, ya que se ha aceptado el beneficio, esto es, las papas, y, por tanto, tiene que pagar el costo, o sea, tiene que pagar maíz. La segunda tarjeta, "Usted no le dio nada a esta persona", no debe ser girada, porque no se ha aceptado el beneficio y, por consiguiente, no es preciso entregar maíz. La tercera tarjeta, "Esta persona le dio a usted maíz", por su parte, tampoco tiene que ser seleccionada, pues ella nos indica que se ha pagado el costo, lo que significa que, haya lo que haya en su lado oculto, ese aldeano no me ha engañado. En cambio, la cuarta tarjeta, "Esta persona no le dio nada a usted", sí que debe ser elegida, puesto que se trata de una persona que no ha pagado el costo y cabe la posibilidad de que se le haya dado el beneficio, es decir, de que haya aceptado papas.

En una segunda versión - la versión con "querer", del problemaFiddick, Cosmides y Tooby (2000) introducen una pequeña modificación: la frase en cursiva que hemos escrito más arriba no aparece y, en su lugar, se le dice al participante que únicamente logra comprender que le dicen

Quiero papas.

y que él sólo es capaz de responder:

Quiero maíz.

Evidentemente, las tarjetas que es necesario girar para resolver adecuadamente esta segunda versión son exactamente las mismas que para la primera versión y por, precisamente, los mismos motivos.

Hemos de decir que, como se nos comenta en el trabajo de Fiddick, Cosmides y Tooby (2000), en las dos versiones, los sujetos se inclinaron mayoritariamente, sin diferencias significativas en los porcentajes, por las tarjetas correctas para descubrir a un posible aldeano tramposo. Esto, naturalmente, fue interpretado como una demostración rotunda y concluyente de que, efectivamente, existen reglas filogenéticas y mecanismos adaptativos en los seres humanos que les posibilitan detectar a los individuos infractores ¿Es este experimento, por consiguiente, una prueba irrefutable de la validez del tipo de formalismo ético que se deduce de la teoría de los contratos sociales? Creemos que no y que estos resultados pueden ser interpretados en un sentido bastante diferente. Veamos cuál es ese sentido. 
Desde nuestra óptica, la primera versión del experimento no demuestra en absoluto la existencia de las pretendidas reglas adaptativas para regular los intercambios sociales, esto es, que las reglas A y B mencionadas anteriormente en realidad guíen el pensamiento humano y ello, sencillamente, porque sus óptimos resultados pueden ser interpretados con facilidad desde una lógica de las proposiciones. El enunciado que el granjero comprende, "Si me da algunas papas, le daré un poco de maíz", es un enunciado condicional y, por consiguiente, es formalizable como $\mathrm{P}=>\mathrm{Q}$. En este sentido, detectar al aldeano que engaña es detectar casos de $\mathrm{P}$ \& no-Q (donde $\mathrm{P}$ corresponde a "Usted le dio papas a esta persona" y no-Q a "Esta persona no le dio a usted nada"). Basta, así, con una simple aplicación del modus tollens para descubrir las tarjetas adecuadas, ya que, en definitiva, como nos muestra la tabla de verdad del condicional, $\mathrm{P} \&$ no-Q es el único caso que hace falso a $\mathrm{P}=>\mathrm{Q}$.

Pero, si esto es así ¿Por qué Fiddick, Cosmides y Tooby (2000) pretenden que su experimento es útil como confirmación de su teoría de los contratos sociales? La respuesta a esta pregunta no es compleja: porque las versiones inciales de WST suelen arrojar pésimos resultados y los participantes en ellas a menudo yerran y no eligen las tarjetas válidas desde el punto de vista lógico, es decir, no seleccionan P \& no-Q. Según Fiddick, Cosmides y Tooby (2000), las versiones iniciales de WST no hacen referencia a situaciones de intercambio, mientras que las suyas sí, y esta diferencia es la que provoca que la ejecución sea distinta en los dos casos, pues en sus experimentos se ven implicados los mecanismos adaptativos que ellos postulan.

Sin embargo, creemos necesario añadir que, entre las versiones iniciales de WST y el experimento 1 de Fiddick, Cosmides y Tooby (2000), existen más diferencias. Las versiones iniciales son, en su gran mayoría, abstractas y carentes de contexto. En ellas, las tarjetas suelen tener un número en una cara y una letra en la otra. Se les muestra a los sujetos, igual que en las versiones de Fiddick, Cosmides y Tooby (2000), cuatro tarjetas, pero lo que figura en cada una de ellas es muy distinto: en la primera generalmente hay una vocal, en la segunda una consonante, en la tercera un número par y en la última un número impar. De esta manera, la labor del sujeto consiste en indicar la(s) tarjeta(s) necesaria(s) para comprobar la verdad o la falsedad de un enunciado que está expresado, casi siempre, en términos similares a éstos

Si en una tarjeta hay una vocal en una cara, entonces, hay un número par en la otra.

En cualquier caso, a nuestro juicio, lo que hay que analizar no es lo que acontece en la versión condicional del experimento 1 de Fiddick, Cosmides y Tooby (2000), ya que en dicha versión no se observa nada anómalo y los participantes eligen la respuesta lógicamente válida. Lo que hay que explicar 


\section{Miguel López Astorga}

es lo que ocurre con las primeras versiones de WST, pues su incorrecta ejecución parece cuestionar la utilización de las leyes lógicas y de las reglas de inferencia formales por parte de los seres humanos. ${ }^{3}$

Sin embargo, lo que nos interesa para este trabajo es que las selecciones de los participantes en la versión condicional del experimento 1 de Fiddick, Cosmides y Tooby (2000) no necesitan, para ser explicadas, de la suposición de ningún mecanismo evolutivo ni de ninguna regla adaptativa adicional, pues son consistentes con el marco de la lógica clásica tradicional y, por ello, no pensamos que demuestren de manera convincente las hipótesis de los teóricos de los contratos sociales referentes a la existencia de reglas en la mente humana para detectar infractores de acuerdos.

Lo que sucede en la segunda versión, la versión con "querer" del experimento, es un poco más complejo, pero no por ese motivo imposible de dilucidar. Desde nuestra óptica, no tiene lugar en la mente del sujeto experimental algo muy diferente a lo que ocurre en la versión condicional. Es cierto que la afirmación "Quiero algunas papas" y la afirmación "Quiero un poco de maíz" no constituyen, las dos juntas, aunque una vaya seguida de la otra, un enunciado condicional formalizable como $\mathrm{P}=>\mathrm{Q}$.

¿Cómo es posible que dos proposiciones separadas ( $\mathrm{P}$ y $Q$ aisladamente) puedan provocar que el individuo las tome como un antecedente y un consecuente unidos por una conectiva condicional? Es fácil responder a esta pregunta a partir de las tesis de Almor y Sloman (2000), ${ }^{4}$ quienes afirman que es posible hablar de dos fases en los individuos que se enfrentan a WST

\section{1.- Construcción de representación.}

2.- Selección de tarjetas en función de la anterior representación.

Los argumentos que Almor y Sloman (2000) presentan para demostrar que estas dos fases realmente tienen lugar se basan en una manipulación de textos procedentes de Gigerenzer y Hug (1992). En dichos textos, se introduce

\footnotetext{
${ }^{3}$ Hemos realizado esta última labor en trabajos anteriores (López Astorga, 2008b). No es, empero, el propósito resolver aquí el problema de las versiones abstractas de WST y, por esta razón, consideramos que es más que suficiente apuntar que, con respecto a este asunto, hemos argumentado que los sujetos habitualmente no comprenden las instrucciones de esas versiones carentes de contexto y que, a menudo, manifiestan la tendencia a interpretar el enunciado no como condicional, sino como conjunción.

${ }^{4}$ El trabajo de Almor y Sloman (2000) puede servirnos para interpretar lo que sucede en esta segunda versión del primer experimento de Fiddick, Cosmides y Tooby (2000), pues, como establecimos (López Astorga, 2004) el enfoque de Almor y Sloman proporciona razones poderosas para sostener que las frases "Quiero algunas papas" y "Quiero un poco de maíz" pueden ser entendidas como los dos términos de un condicional, lo cual significa que pueden permitirnos llegar al enunciado "Si me das papas, entonces, te daré maíz".
} 
una incoherencia entre la historia narrada y la regla condicional a comprobar. Igualmente, incorporan a sus versiones de WST un elemento novedoso y nada frecuente en las investigaciones que sobre esta tarea suelen ofrecer los investigadores del ámbito de la ciencia cognitiva: solicitan a los participantes en el experimento que, una vez realizada su labor, intenten recordar la regla que tenían que revisar.

A título ilustrativo, podemos reflejar que en una de sus condiciones experimentales sus participantes tuvieron que imaginar que trabajaban para una empresa que había prometido que todos aquellos empleados que trabajaran durante el fin de semana serían compensados con un día libre durante la semana. Sin embargo, como existían rumores de que esta promesa no solía cumplirse, deseaban comprobar si, realmente, aquellos trabajadores que sacrificaban un fin de semana obtenían su premio. Una regla para esta versión debería ser semejante a ésta

A) Si trabaja el fin de semana, entonces, se le concede un día libre.

Pero Almor y Sloman (2000) propusieron a algunos sujetos experimentales esta otra regla en la que el antecedente y el consecuente se encuentran rotados

B) Si se le concede un día libre, entonces, debe haber trabajado el fin de semana.

Lo que descubrieron Almor y Sloman (2000) es que estos participantes no recordaban la regla literal que aparecía en el problema (B), sino una regla coherente con la historia narrada (A). Además, notaron que se daba una tendencia significativa a seleccionar las tarjetas válidas desde el punto de vista lógico para (A), y no para (B). De este modo, su conclusión apuntó a la idea de que las personas no procesan necesariamente la información como les llega, sino de una manera en que sea consistente con el resto de sus representaciones.

Si a esta tesis de Almor y Sloman (2000) les añadimos que, como nos cuenta Deaño (1999), a cada conectiva en lenguaje formal no le corresponde una única expresión en lenguaje ordinario, como tampoco tienen una traducción al lenguaje artificial de la lógica todas las conexiones del lenguaje natural, no nos resulta difícil entender por qué las dos proposiciones separadas de la versión con "querer" del experimento 1 de Fiddick, Cosmides y Tooby (2000) pueden ser interpretadas como los dos términos, antecedente y consecuente, de una relación condicional.

De acuerdo con la lógica de proposiciones, las selecciones de los participantes en esa versión del experimento 1 de Fiddick, Cosmides y Tooby (2000) son las correctas para un enunciado condicional, por ejemplo, son las 
que corresponden a $\mathrm{P} \&$ no-Q. Por ello, no nos parece demasiado artificioso pensar que, a causa del contexto descrito, los sujetos experimentales se construyen una representación mental (primera fase de procesamiento de Almor y Sloman, 2000) más o menos semejante a "Si me das papas, entonces te daré maíz". El escenario es el de un individuo que desea intercambiar sus papas por maíz. Él ofrece papas y a él le presentan maíz ¿Cómo no llegar, ante tal situación, a la conclusión de que lo que hay que comprobar es una regla parecida a "Si papas, entonces maíz"?

Todas estas reflexiones nos conducen a poner en duda los postulados fundamentales de los defensores de la teoría de los contratos sociales. En nuestra opinión, el experimento 1 de Fiddick, Cosmides y Tooby (2000) no muestra ningún fenómeno que la tradicional lógica de proposiciones no pueda explicar. Lo único que evidencia dicho experimento, a nuestro juicio, es que siguen siendo bastante desconocidos, para la ciencia cognitiva, los procedimientos por medio de los cuales los seres humanos formalizan o traducen el lenguaje natural en lenguaje formal. Y es que, asumiendo que las posibilidades de procesamiento del lenguaje natural son diversas y que no se encuentran determinadas (aunque sí, por supuesto, hasta cierto punto, condicionadas) por las expresiones literales utilizadas en los textos, se torna obvio que, en realidad, las diferencias entre la versión condicional y la versión con "querer" del experimento 1 de Fiddick, Cosmides y Tooby (2000) son únicamente aparentes y simplemente estéticas.

Por consiguiente, creemos que la teoría de los contratos sociales necesita apoyos experimentales más fuertes que los que hemos analizado en este apartado, ya que parece que no es tan sencillo demostrar "científicamente" este nuevo formalismo ético. En definitiva, esta teoría sólo es uno de tantos enfoques que se han presentado con la intención de explicar los extraños resultados de WST y, sin ir más lejos, en el trabajo de Fiddick, Cosmides y Tooby (2000) se polemiza continuamente con otra propuesta alternativa rival: la de la teoría de la relevancia, la cual se puede encontrar expuesta en sus líneas fundamentales en Sperber, Cara y Girotto (1995). Sin duda, la teoría de la relevancia es también bastante sugerente y atractiva en sus argumentaciones, pero no es el objeto de este estudio y, por ello, no acometemos aquí su análisis. Sólo la nombramos con el propósito de constatar que no existe un acuerdo teórico con respecto a WST y que el planteamiento de los contratos sociales no es, necesariamente, el más aceptado en el ámbito de la psicología del razonamiento.

\section{EL PROBLEMA DE LA CABAÑA}

Hemos hecho mención al hecho de que son muy diversos los experimentos que ofrece la literatura con el objetivo de evidenciar la 
presencia de reglas adaptativas de contrato social en las personas, es decir, de reglas como las A y B ya indicadas para controlar los intercambios y los acuerdos. Como hemos establecido, el experimento 1 de Fiddick, Cosmides y Tooby (2000) es lo bastante representativo de este enfoque teórico y, por consiguiente, la revisión crítica que hemos realizado de él es aplicable a la mayor parte de la experimentación que han presentado sus defensores. No obstante, deseamos mostrar también lo que sucede en determinados trabajos experimentales igualmente asociados a esta teoría en los que intervienen otras variables. Con tal fin, hemos seleccionado otro artículo característico de esta corriente psicológica de pensamiento, el cual nos va a permitir constatar de nuevo que los apoyos que la propuesta de los contratos sociales suele presentar no son concluyentes ni nos llevan forzosamente a aceptar sus supuestos.

El trabajo seleccionado es el de Gigerenzer y Hug (1992), quienes argumentan que no basta para que una versión de WST obtenga buenos resultados con que un contrato social pueda ser deducido de su regla, sino que es preciso que explícita y claramente se pida en las instrucciones que se localicen incumplidores de tal contrato, esto es, personas que toman el beneficio sin asumir el costo. Ellos tratan de apoyar esta hipótesis con sus experimentos, en los que se cotejan versiones de WST con la misma regla de contrato social, pero con diferente contexto. De esta manera, los dos contextos que proporcionan se traducen en dos tipos de versiones: versión "tramposo" (Cheating version) que solicita hallar, como su propio nombre lo indica, individuos tramposos, y versión "no tramposo" (No cheating version) que pide también localizar infractores, pero con el objetivo de descubrir si la regla de contrato social se halla vigente o no. Vamos a analizar a continuación la historia de Gigerenzer y Hug (1992) que parece llamar más la atención de Fiddick, Cosmides y Tooby (2000): el problema de la cabaña.

La versión "tramposo" del problema de la cabaña establece una regla en el Club Alpino Suizo para el disfrute de su cabaña: "si se pasa una noche en la cabaña, entonces hay que transportar un cargamento de leña desde el valle". Evidentemente, la tarea aquí es detectar individuos que utilizan la cabaña indebidamente, es decir, individuos que, sin cargar leña, pernoctan en la cabaña (P \& no-Q).

Pero la versión "no tramposo" amplía el contexto. Nos habla de un turista alemán que observa que diferentes personas llevan leña a la cabaña del Club Alpino Suizo y que se pregunta si existirá una norma para pasar una noche en dicha cabaña, una norma idéntica a la enunciada en la versión "tramposo". No obstante, se baraja también la posibilidad de que no haya tal norma y de que los miembros del Club Alpino Suizo transporten leña a la cabaña de modo altruista. El sujeto experimental tiene que suponer, sin 
embargo, que la regla verdaderamente existe y buscar infractores a la misma, si bien el propósito, como se puede comprobar, no es el mismo que en la versión anterior, pues se admite como hipótesis que la regla sea una simple suposición.

Los porcentajes de selección fueron mucho mejores en la versión "tramposo" (se registró casi el doble de elecciones válidas que en la versión "no tramposo"), Gigerenzer y Hug consideraron esta circunstancia como un apoyo indiscutible para sus tesis. No obstante, desde nuestra óptica, es posible explicar también estos hechos sin recurrir a mecanismos evolutivos adaptativos. Pensamos que no es necesario decir mucho sobre la versión "tramposo" y que en ella, verdaderamente, no hay ningún problema que solucionar, ya que sucede lo mismo que en el experimento 1 de Fiddick, Cosmides y Tooby (2000): los sujetos eligen las tarjetas correctas desde el punto de vista lógico. Las dificultades se hallan, en nuestra opinión, en la versión "no tramposo", si bien tales dificultades pueden disiparse si tenemos en cuenta varios puntos.

En la versión "no tramposo" interviene un número mayor de variables $\mathrm{y}$, por consiguiente, el participante en ella tiene que procesar más información (López Astorga, 2004). Ambas versiones no requieren la misma actividad cognitiva, lo que significa que, desde el punto de vista gnoseológico, no conducen forzosamente a las mismas representaciones mentales. Además, ya Yachanin y Tweney (1982) señalaron que el que en muchas versiones de WST no se les pida lo mismo a los participantes influye en su ejecución y, por tanto, en su selección. En concreto, Yachanin y Tweney se refieren explícitamente, como a uno de los factores diferenciadores que determinan la elección de tarjetas, al hecho de que a veces se presenta la regla como cierta, como en la versión "tramposo", y en otras ocasiones como hipotética, como en la versión "no tramposo". Esta circunstancia ha pasado inadvertida para muchos autores que han construido versiones de WST, pero, según Yachanin y Tweney, es crucial en los resultados de la tarea.

Ante esto, el aspecto más importante a tener en cuenta es que no es, según creemos, legítimo comparar los resultados de las dos versiones del problema de la cabaña de Gigerenzer y Hug (1992), ello porque son dos tareas completamente distintas que sólo comparten un escenario similar. No es lo mismo lo que se le pide al sujeto experimental en los dos casos y, lo más importante, tampoco es la misma la respuesta correcta en los dos ejercicios. Por cierto, la elección lógicamente válida para la versión "tramposo" es la combinación P y no-Q, pero ésa no es la respuesta correcta para la versión "no tramposo", pues esta última, por su parte, al no ser en su contexto ninguna tarjeta absolutamente concluyente, no tiene, en realidad, una respuesta válida definida. En ella, se les está solicitando a los participantes que realicen una 
tarea imposible en la que no puede deducirse, de ninguna manera, si la regla expuesta ("si se pasa una noche en la cabaña, entonces hay que transportar un cargamento de leña desde el valle") es verdadera o falsa, una tarea que no puede resolverse ni girando las cuatro tarjetas, y, ante ello, ninguna conclusión se puede obtener cotejando los resultados de ambas versiones. Y es que las representaciones mentales que la versión "no tramposo" permite al sujeto construirse pueden conducirle por derroteros insospechados para el investigador. Pero vamos a analizar cada tarjeta por separado para demostrar lo que estamos defendiendo, esto es, que la versión "no tramposo" no tiene una respuesta correcta.

Si el participante elige la tarjeta relativa a pasar una noche en la cabaña (P), no le será muy informativo lo que encuentre en su lado oculto. Si encuentra que se trae leña (Q), ello puede ser casual o, sencillamente, un gesto de buena voluntad. Por el contrario, si halla que no se trae (no-Q), tampoco eso indica que no exista la regla, porque podemos estar ante un infractor.

La tarjeta referente a no pasar la noche en la cabaña (no-P) continúa sin aportar demasiado. Si va acompañada de que no trae leña (no-Q) no significa nada, pues el individuo en cuestión no tiene ninguna obligación. Si aparece que trae leña $(\mathrm{Q})$, tampoco ésa sería una información decisiva, ya que, aunque podría tratarse de un miembro local del Club que trae leña (segunda posibilidad en la versión "no tramposo"), también podría ser un sujeto no perteneciente al Club que realiza la labor voluntariamente.

La tarjeta correspondiente a traer leña (Q) sigue sin ser concluyente. Si se trata de un sujeto que pasa la noche en la cabaña $(\mathrm{P})$, sigue sin quedar claro si estamos ante un gesto de buena voluntad o ante una obligación. Si no pasa la noche en la cabaña (no-P), igual que en la tarjeta anterior, no podemos saber si ese individuo es un miembro local (segunda posibilidad) o una persona que ha querido colaborar sin esperar nada a cambio.

Por último, la tarjeta relativa a no traer leña (no-Q) también se encuentra revestida de la misma indeterminación que las anteriores. Si en su lado oculto presenta que el sujeto pasó la noche en la cabaña (P), desconoceríamos si ese sujeto es un infractor o si lo que ocurre es que la regla no existe. Si figura que no pasó la noche en la cabaña (no-P), estamos ante un individuo que no tiene que pagar ningún costo por ningún beneficio.

Por tanto, esta versión de WST es, sin más, irrealizable y no es, por ello, apropiada para comparar sus resultados con los de la versión "tramposo". Estamos ante dos tareas similares en la forma, pero bastante diferentes en su ejecución. En la segunda versión, para saber si la regla existe con total seguridad, lo único que se puede hacer es preguntar a la autoridad correspondiente o responsable del Club Alpino Suizo, pero, evidentemente, esa posibilidad no está a disposición del participante. 


\section{Miguel López Astorga}

Contra esta argumentación nuestra, se podría objetar, está claro, que en la versión "no tramposo", a pesar de que no se conoce si la norma efectivamente está establecida o no, se le pide al sujeto experimental igualmente que trate de hallar violadores de la misma, lo cual sí es una labor viable y no es hecho por la mayoría de los participantes. No obstante, creemos que replicar a esta posible objeción no es complejo. Por una parte, podemos insistir en que en esta segunda versión del problema de la cabaña de Gigerenzer y Hug (1992) hay mucha más información que en la primera y en que el participante puede no entender nítidamente todas las instrucciones. Por otra parte, podemos también presentar otra reflexión aún más contundente: si la hipótesis de Gigerenzer y Hug es que lo verdaderamente importante para que se activen los mecanismos de contrato social es la indicación explícita de buscar tramposos y en la segunda versión de su problema de la cabaña se da tal indicación ¿Por qué, entonces, sus sujetos no seleccionan las tarjetas adecuadas? Pensamos que, lejos de favorecer esta objeción al planteamiento de Gigerenzer y Hug, le haría un daño todavía mayor.

Lo máximo que pueden hacer los sujetos experimentales en la versión "no tramposo" es intentar, por inducción, generalizar en base a casos concretos, operar a partir de una conjunción y procurar encontrar casos de, por ejemplo, P \& no-Q. Por supuesto, los resultados logrados de este modo nunca serían definitivos, pero hemos de tener presente que, en estas circunstancias, los individuos sólo pueden hacer aquello que consideran que les puede proporcionar una mayor información, aunque ésta sea únicamente aproximada.

Por todo esto, podemos decir que el problema de la cabaña, debido a la falta de correspondencia entre sus dos versiones, no prueba la existencia de mecanismos adaptativos o de reglas evolutivas de contrato social.

\section{CONCLUSIONES}

Hemos seleccionado dos ejemplos emblemáticos de trabajos experimentales que se han utilizado en la literatura para intentar demostrar que existen mecanismos evolutivos y reglas adaptativas en los seres humanos cuya función es regular sus intercambios sociales. Sin embargo, después de revisar críticamente los resultados de tales trabajos, llegamos al convencimiento de que pueden ser fácilmente interpretados sin necesidad de recurrir a ninguna estructura mental específica en la mente de las personas.

Así, si los seres humanos disponemos de reglas en nuestro entendimiento para descubrir a los sujetos que no cumplen los acuerdos, ello sólo es, desde nuestro punto de vista, una hipótesis hasta el momento presente. Si los defensores de los contratos sociales quieren demostrar que sus supuestos realmente se sostienen, tienen forzosamente que buscar evidencias 
en pruebas experimentales de mayor rigor. Como indicamos al comienzo de este trabajo, la tesis de fondo en la que se basa esta teoría es que en la arquitectura cognitiva humana hay dominios de contenido específico y evolucionados que funcionan como motores de inferencia y que se suman a las competencias inferenciales de contenido general que puedan existir. Nosotros entendemos que, al aludir a competencias inferenciales de contenido general, la propuesta de los contratos sociales se refiere a las capacidades de pensamiento lógico formal abstracto de las que se habla, por ejemplo, en Beth y Piaget (1966). De este modo, lo que nos planteamos es por qué se precisa recurrir a dominios específicos adicionales para explicar lo que ya puede ser comprendido con las reglas de inferencia de la lógica clásica. Por supuesto, nos parece muy interesante y enormemente positivo que se pretenda investigar y avanzar en el conocimiento de las estructuras cognitivas humanas, pues puede que no sea apropiado quedarse estancado en el marco lógico formal del que ya disponemos y aceptarlo como definitivo e inamovible. Sin embargo, tampoco parece aconsejable rechazarlo, sin más, por el simple hecho de que no nos complazca la imagen de un ser humano que razona y realiza sus inferencias a partir de leyes mentales con correspondencias más o menos directas con los principios lógicos tradicionales. Los defensores de la teoría de los contratos sociales no descartan, es cierto, la inferencia lógica, pero recurren a mecanismos y reglas complementarias que, a nuestro juicio, no son necesarias para dar cuenta de los fenómenos implicados en los ejercicios que ellos proponen a sus sujetos experimentales.

En nuestra opinión, los teóricos de este contractualismo psicológico contemporáneo parten de una idea definida de lo que es la naturaleza humana, lo cual es absolutamente legítimo. Lo que nos cuesta admitir es su pretensión relativa a que su enfoque se valida científicamente a partir de sus trabajos experimentales. $\mathrm{Y}$ es que hemos establecido en páginas precedentes que tales trabajos no son interpretables de una manera unívoca y que no quedan demostrados sus supuestos. Por ello, continúa siendo igualmente legítimo argumentar teóricamente contra sus tesis y contra sus consecuencias implicadas.

Lo dicho provoca que encontremos justificada una lectura de los planteamientos de la teoría de los contratos sociales como la que hicimos al comienzo, por ejemplo, en cuanto un nuevo formalismo ético, el cual, claro está, puede polemizar con otras teorías éticas del presente y de la historia del pensamiento, pero también puede ser puesto en cuestión por medio de procedimientos propios y característicos de la especulación filosófica.

El caso es que el poder seductor de la propuesta de los contratos sociales es tremendo, puesto que — ya lo indicamos más arriba— habla de un puente de comunicación entre la lógica y la ética, un puente cuyo pilar básico es una cierta forma especial de razonar en el ámbito de los intercambios y de 
los negocios humanos. Empero, insistimos en que, para que pueda sostenerse de un modo indiscutible la existencia de esa forma especial de razonamiento, es necesario contar con bases más sólidas. Somos conscientes de que exactamente la misma crítica se puede presentar contra un enfoque en la línea de Beth y Piaget que, implícita o explícitamente, considere que las leyes del pensamiento coinciden con las de la lógica. Con respecto a esto último, hemos de aclarar que no suscribimos una posición en esa línea. Lo que nosotros argumentamos es que, mientras contemos con una explicación sencilla y, al mismo tiempo, completa de un problema, no resulta muy provechoso o afortunado buscar recursos más sofisticados que, inevitablemente, van a conducirnos a una interpretación más compleja.

Es obvio que también nosotros tenemos nuestra visión personal de lo que es la naturaleza humana. Deseamos expresar, sobre ella, que de este trabajo no debe deducirse que concebimos a la persona como un ser provisto de una mente capaz de efectuar inferencias de un modo rígido y exacto, tal y como lo exigen las leyes y las reglas de la lógica formal. Consideramos que son múltiples los factores (culturales, académicos, personales) que pueden tener incidencia en las actividades intelectivas humanas y que en los procesos mentales intervienen también los ámbitos emocionales y pasionales. Ahora bien, aceptar esto no es incompatible con admitir que la lógica es un elemento característico más de la esencia de la persona, porque, para defender el papel que desempeñan otros elementos en su comportamiento, no es necesario rechazarla u obviarla. De hecho, ni siquiera la teoría de los contratos sociales cuestiona su validez, pues señala claramente que los mecanismos evolutivos adaptativos se suman a las capacidades generales que ya puedan tener los sujetos.

Universidad de Los Lagos*

Departamento de Educación

Avda. Alcalde Fuchslocher 1305

Osorno (Chile)

m.lopez@ulagos.cl 
Los contratos sociales

\section{BIBLIOGRAFÍA}

ALMOR, A.; SLOMAN, S. A. "Reasoning versus Text Processing in the Wason Selection Task -A Non-Deontic Perspective on Perspective Effects", en Memory \& Cognition 28 (2000):1060-1069.

BARKOW, J.; COSMIDES, L.; TOOBY, J. (Eds.). The Adapted Mind: Evolutionary Psychology and the Generation of Culture. Nueva York: Oxford University Press, 1992.

BETH, E. W.; PIAGET, J. Mathematical Epistemology and Psychology. Dordrecht: Reidel, 1966.

COSMIDES, L. "The Logic of Social Exchange: Has Natural Selection Shaped How Humans Reason? Studies with the Wason Selection Task". Cognition 31 (1989):187-276.

COSMIDES, L.; TOOBY, J. "Are Humans Good Intuitive Statisticians after All? Rethinking some Conclusions of the Literature on Judgment under Uncertainty". Cognition 58 (1996):1-73.

------ "A Logical Design for the Mind?" Contemporary Psychology 41(5) (1996b):448-450.

DEAÑO, A. Introducción a la Lógica Formal. Madrid: Alianza, 1999.

FIDDICK, L.; COSMIDES, L.; TOOBY, J. "No Interpretation without Representation: The Role of Domain-Specific Representations and Inferences in the Wason Selection Task". Cognition 75 (2000):1-79.

GIGERENZER, G.; HOFFRAGE, U. "How to Improve Bayesian Reasoning without Instruction: Frequency Formats". Psychological Review 102 (1995):684-704.

GIGERENZER, G.; HOFFRAGE, U.; KLEINBOLTING, H. "Probabilistic Mental Models: A Brunswikian Theory of Confidence". Psychological Review 98 (1991):506-528.

GIGERENZER, G.; HUG, K. "Domain-Specific Reasoning: Social Contratcs, Cheating and Perspective Change". Cognition 43 (1992):127-171.

GIGERENZER, G.; TODD, P.; The Abc Research Group. Simple Heuristic that Make us Smart. Nueva York: Oxford University Press, 1999.

LÓPEZ ASTORGA, M. Revisión del Razonamiento Condicional a partir de la Tarea de Selección. Revising Conditional Reasoning by means of Selection Task (Spanish text). Ann Arbor (Michigan): Proquest, Information and Learning Company, 2004.

------ "Las Cuatro Tarjetas y el Razonamiento Humano", en Ciencia Cognitiva: Revista Electrónica de Divulgación 2:3 (2008):78-80.

"Tarea de Selección: Una Explicación desde la Lógica Formal", en A Parte Rei. Revista de Filosofía 59 (2008b).

http://serbal.pntic.mec.es/ cmunoz11/astorga59.pdf. (Consultado: 10 de mayo de 2010).

SANTAMARÍA, C. Introducción al Razonamiento Humano. Madrid: Alianza, 1995. 


\section{Miguel López Astorga}

SPERBER, D. "The Modularity of Thought and the Epidemiology of Representations". L. Hirschfeld y S. Gelman (Eds.). Mapping the Mind: Domain Specificity in Cognition and Culture. Nueva York: Cambridge University Press, 1994.

SPERBER, D.; CARA, F.; GIROTTO, V. "Relevance Theory Explains the Selection Task". D. Sperber (Ed.). Metarepresentation. Nueva York: Vancouver Studies in Cognitive Science. Oxford University Press, 1995.

WASON, P. C. "Reasoning". B. Foss (Comp.). New Horizons in Psychology. H. Middlesex: Penguin, 1966.

YACHANIN, S. A.; TWENEY, R. D. "The Effect of Thematic Content on Cognitive Strategies in the Four-Card Selection Task". Bulletin of Psychonomic Society 19 (1982):87-90. 